\title{
Accuracy and precision of two methods of blood lead estimation
}

\author{
A. J. FOX and M. H. P. SAYERS \\ Employment Medical Advisory Service Division, Department of Employment, London W2
}

In recent years, increasing reliance has been placed upon the blood lead as a measurement of occupational exposure. The aim is to limit absorption before biochemical mechanisms are interfered with significantly. In the present state of knowledge, there is general agreement that a blood lead below $80 \mu \mathrm{g} / 100 \mathrm{ml}$ rarely results in harmful effects, but the aim should be to strive for lower quantities than this to ensure that, ultimately, the lead worker is at no greater hazard than the ordinary citizen.

This increasing use of the blood lead has been made possible by the development of micro techniques using atomic absorption spectrophotometry. There is ample evidence (see, for example, White, 1968; Delves, 1970; and Cernik and Sayers, 1971) to show that these new methods can be as precise as established analytical procedures. The coefficient of variation is of the order of $5 \%$ in the single estimate. The standard deviation can, however, be reduced by reporting on the mean of several estimates. With most macro techniques, this is too time-consuming and expensive, but using micro methods, replication is comparatively simple. The variance of the difference between results obtained by two different methods is the sum of their individual variances. A $95 \%$ confidence interval would suggest that the difference between two individual results should lie within $\pm 14 \%$ ( 2 standard deviations).

In order to demonstrate the differences that might be observed, the results of a recent investigation into the accuracy of the two routine methods of blood lead estimation used by this laboratory, viz., cathode ray polarography and atomic absorption spectrophotometry, are summarized in the Figure. Over the course of the last few months, the blood lead has been routinely estimated from venous blood samples by the atomic absorption spectrophotometry micromethod (Cernik and Sayers, 1971) using a $6.5 \mathrm{~mm}$ punched disc. The blood lead is reported as the mean of two estimates (i.e., two discs are analysed). As a routine check, every twentieth sample is also analysed by polarography (Cernik, 1967). For the purpose of this exercise, however, these samples were also duplicated so that the variances of the two methods

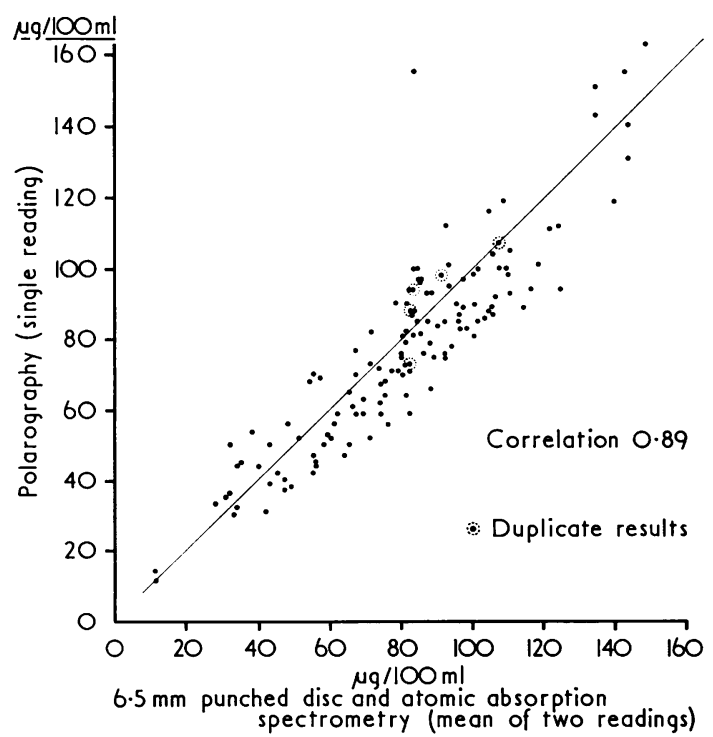

FIGURE. A comparison of results reported by two routine methods of estimating quantities of lead in venous blood samples (141 samples). 
might be estimated. Since the reported result by polarography is usually based on a single reading, in the Figure the first reading estimated by polarography is compared with the mean found by the punched disc method. The standard deviations of the two methods of reporting are 4.15 for polarography and 4.66 for atomic absorption spectrophotometry, which are equivalent for practical purposes.

The Figure shows the relationship between the results, assuming that the first estimate obtained by polarography would be the one reported. The two regression lines obtained are:

$$
\begin{aligned}
& \mathrm{PD}=0.86 \times \mathrm{POL}+13.61 \text {, and } \\
& \mathrm{POL}=0.91 \times \mathrm{PD}+4.07
\end{aligned}
$$

and the correlation is +0.89 , where PD and POL represent the results by the punched disc and polarographic methods respectively.

Given the range of values over which we sampled and the estimated variances of the two methods, the estimated expected correlation is approximately +0.92 . Clearly, the correlation observed, +0.89 , suggests that the results obtained are no less accurate over the range of values considered than one would expect comparing two methods with similar inbuilt variances. There is, therefore, no suggestion of bias between the two methods, and the differences observed are likely to be due to chance.

These results show that the standard deviation of the difference of results done by two or more methods added to the effects of sampling errors will account for some of the anomalies between individual results reported by different laboratories. These discrepancies often confuse the clinician responsible for interpretation and if in doubt he is well advised to repeat the investigation on a fresh blood sample.

In an attempt to standardize the blood lead estimates reported by different laboratories, a routine National Quality Control scheme has recently been set up in co-operation with the Wolfson Research Laboratory at the Queen Elizabeth Medical Centre, Edgbaston, Birmingham. A specially prepared haemolysed, sterile sample of blood is sent to over 50 participating laboratories. Results are subsequently analysed to give the overall mean, standard deviation, and histogram indicating where the result returned by each laboratory lies. Further analysis is undertaken according to the method of estimation. Results of the early trials reveal a wide variation between laboratories. This exercise is continuing on a monthly basis.

\section{References}

Cernik, A. A. (1967). A dry ashing method for the determination of blood lead using cathode ray polarography. British Journal of Industrial Medicine, 24, 289-293.

- , and Sayers, M. H. P. (1971). Determination of lead in capillary blood using a paper punched disc atomic absorption technique. British Journal of Industrial Medicine, 28, 392-398.

Delves, H. T. (1970). A micro-sampling method for the rapid determination of lead in blood by atomic-absorption spectrophotometry. Analyst, 95, 431-438.

White, R. A. (1968). B.N.F.M.R.A. Research Report A.1707 and paper presented at the International Atomic Absorption Spectroscopy Conference, Sheffield, 1969.

Received for publication February 2, 1973. Accepted for publication April 6, 1973. 